\title{
Nonlinear cyclic weak contractions in G-metric spaces and applications to boundary value problems
}

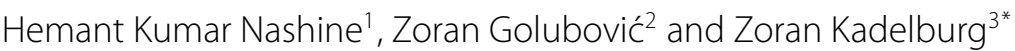

${ }^{\text {*Correspondence: }}$

kadelbur@matf.bg.ac.rs

${ }^{3}$ Faculty of Mathematics, University

of Belgrade, Studentski trg 16,

Beograd, 11000, Serbia

Full list of author information is

available at the end of the article

\begin{abstract}
We present fixed point theorems for nonlinear cyclic mappings under a generalized weakly contractive condition in $G$-metric spaces. We furnish examples to demonstrate the usage of the results and produce an application to second-order periodic boundary value problems for ODEs.

MSC: $47 \mathrm{H} 10 ; 34 \mathrm{~B} 15$
\end{abstract}

Keywords: cyclic contraction; fixed point; G-metric space; weakly contractive condition; altering distance function; boundary value problem

\section{Introduction}

Nonlinear analysis is a remarkable confluence of topology, analysis and applied mathematics. The fixed point theory is one of the most rapidly growing topics of nonlinear functional analysis. It is a vast and inter-disciplinary subject whose study belongs to several mathematical domains such as classical analysis, differential and integral equations, functional analysis, operator theory, topology and algebraic topology etc. Most important nonlinear problems of applied mathematics reduce to finding solutions of nonlinear functional equations (e.g., nonlinear integral equations, boundary value problems for nonlinear ordinary or partial differential equations, the existence of periodic solutions of nonlinear partial differential equations). They can be formulated in terms of finding the fixed points of a given nonlinear mapping on an infinite dimensional function space into itself.

In 1922, Banach's contraction mapping principle appeared [1] and it was known for its simple and elegant proof by using the Picard iteration. If $(X, d)$ is a complete metric space and $T: X \rightarrow X$ is a contraction, that is, there exists $k \in(0,1)$ such that

$$
d(T x, T y) \leq k d(x, y), \quad \forall x, y \in X
$$

the conclusion is that $T$ has a fixed point, in fact, exactly one of them.

The simplicity of its proof and the possibility of attaining the fixed point by using successive approximations let this theorem become a very useful tool in analysis and its applications. There is a great number of generalizations of the Banach contraction principle in the literature (see, e.g., [2] and the references cited therein).

It is important to note that the inequality (1.1) implies the continuity of $T$. A natural question is whether we can find contractive conditions which will imply the existence

(c) 2012 Nashine et al.; licensee Springer. This is an Open Access article distributed under the terms of the Creative Commons Attribution License (http://creativecommons.org/licenses/by/2.0), which permits unrestricted use, distribution, and reproduction in any medium, provided the original work is properly cited. 
of a fixed point in a complete metric space but will not imply continuity. The question was answered by Kirk et al. [3] and turned the area of investigation of fixed points by introducing cyclic representations and cyclic contractions, which can be stated as follows.

Definition 1 [3] Let $(X, d)$ be a metric space. Let $p$ be a positive integer, $\mathcal{A}_{1}, \mathcal{A}_{2}, \ldots, \mathcal{A}_{p}$ be subsets of $X, Y=\bigcup_{i=1}^{p} \mathcal{A}_{i}$ and $T: Y \rightarrow Y$. Then $Y$ is said to be a cyclic representation of $Y$ with respect to $T$ if

(i) $\mathcal{A}_{i}, i=1,2, \ldots, p$ are nonempty closed sets, and

(ii) $T\left(\mathcal{A}_{1}\right) \subseteq \mathcal{A}_{2}, \ldots, T\left(\mathcal{A}_{p-1}\right) \subseteq \mathcal{A}_{p}, T\left(\mathcal{A}_{p}\right) \subseteq \mathcal{A}_{1}$.

Moreover, $T$ is called a cyclic contraction if there exists $k \in(0,1)$ such that $d(T x, T y) \leq$ $k d(x, y)$ for all $x \in \mathcal{A}_{i}$ and $y \in \mathcal{A}_{i+1}$, with $\mathcal{A}_{p+1}=\mathcal{A}_{1}$.

Notice that although a contraction is continuous, a cyclic contraction need not to be. This is one of the important gains of this notion. Kirk et al. obtained, among others, cyclic versions of the Banach contraction principle [1], of the Boyd and Wong fixed point theorem [4] and of the Caristi fixed point theorem [5]. Following the paper [3], a number of fixed point theorems on cyclic representation with respect to a self-mapping have appeared (see, e.g., [6-13]).

On the other hand, in 2006, Mustafa and Sims [14, 15] introduced the notion of generalized metric spaces or simply $G$-metric spaces. Several authors studied these spaces a lot and obtained several fixed and common fixed point theorems (see, e.g., [16-26]).

Khan et al. [27] introduced the concept of an altering distance function.

Definition 2 [27] A function $\psi:[0,+\infty) \rightarrow[0,+\infty)$ is called an altering distance function if the following properties are satisfied:

(i) $\psi$ is continuous and non-decreasing,

(ii) $\psi(t)=0$ if and only if $t=0$.

They proved the following theorem.

Theorem 1 [27] Let $(X, d)$ be a complete metric space, let $\varphi$ be an altering distance function, and let $T: X \rightarrow X$ be a self-mapping which satisfies the following inequality:

$$
\varphi(d(T x, T y)) \leq c \varphi(d(x, y))
$$

for all $x, y \in X$ and for some $0<c<1$. Then $T$ has a unique fixed point.

Putting $\varphi(t)=t$ in the previous theorem, (1.2) reduces to (1.1).

Rhoades [28] extended Banach's principle by introducing weakly contractive mappings in complete metric spaces.

Definition 3 [28] Let $(X, d)$ be a metric space. A mapping $T: X \rightarrow X$ is called weakly contractive if

$$
d(T x, T y) \leq d(x, y)-\varphi(d(x, y)), \quad \forall x, y \in X,
$$

where $\varphi$ is an altering distance function.

He proved the following result. 
Theorem 2 [28] Let $(X, d)$ be a complete metric space. If $T: X \rightarrow X$ is a weakly contractive mapping, then $T$ has a unique fixed point.

If one takes $\varphi(t)=(1-k) t$, where $0<k<1$, then (1.3) reduces to (1.1).

Dutta and Choudhury obtained in [29] the following generalization of Theorems 1 and 2.

Theorem 3 [29] Let $(X, d)$ be a complete metric space and let $T: X \rightarrow X$ satisfy

$$
\psi(d(T x, T y)) \leq \psi(d(x, y))-\varphi(d(x, y))
$$

for all $x, y \in X$, where $\psi$ and $\varphi$ are altering distance functions. Then $T$ has a unique fixed point.

Weak inequalities of the above type have been used to establish fixed point results in a number of subsequent works, some of which are noted in [2,30-35] and the references cited therein.

In the present paper, we introduce nonlinear cyclic contraction mappings under a generalized weakly contraction condition in G-metric spaces. It is followed by the proof of existence and uniqueness of fixed points for such mappings. The obtained result generalizes and improves many existing theorems in the literature. Some examples are given in support of our results. In conclusion, we apply accomplished fixed point results for generalized cyclic contraction type mappings to the study of existence and uniqueness of solutions for a class of second-order periodic boundary value problems for ODEs.

\section{Preliminaries}

For more details on the following definitions and results, we refer the reader to [15].

Definition 4 Let $X$ be a nonempty set and let $G: X \times X \times X \rightarrow \mathbb{R}^{+}$be a function satisfying the following properties:

(G1) $G(x, y, z)=0$ if $x=y=z$;

(G2) $0<G(x, x, y)$ for all $x, y \in X$ with $x \neq y$;

(G3) $G(x, x, y) \leq G(x, y, z)$ for all $x, y, z \in X$ with $z \neq y$;

(G4) $G(x, y, z)=G(x, z, y)=G(y, z, x)=\cdots$ (symmetry in all three variables);

(G5) $G(x, y, z) \leq G(x, a, a)+G(a, y, z)$ for all $x, y, z, a \in X$ (rectangle inequality).

Then the function $G$ is called a $G$-metric on $X$ and the pair $(X, G)$ is called a $G$-metric space.

Note that it can be easily deduced from (G4) and (G5) that

$$
|G(x, y, y)-G(x, z, z)| \leq 2 G(y, z, z)
$$

holds for all $x, y, z \in X$.

Definition 5 Let $(X, G)$ be a $G$-metric space and let $\left(x_{n}\right)$ be a sequence of points in $X$.

1. A point $x \in X$ is said to be the limit of the sequence $\left(x_{n}\right)$ if $\lim _{n, m \rightarrow \infty} G\left(x, x_{n}, x_{m}\right)=0$, and one says that the sequence $\left(x_{n}\right)$ is $G$-convergent to $x$. 
2. The sequence $\left(x_{n}\right)$ is said to be a G-Cauchy sequence if for every $\varepsilon>0$, there is a positive integer $N$ such that $G\left(x_{n}, x_{m}, x_{l}\right)<\varepsilon$ for all $n, m, l \geq N$; that is, if $G\left(x_{n}, x_{m}, x_{l}\right) \rightarrow 0$ as $n, m, l \rightarrow \infty$.

3. $(X, G)$ is said to be $G$-complete (or a complete $G$-metric space) if every $G$-Cauchy sequence in $(X, G)$ is $G$-convergent in $X$.

Thus, if $x_{n} \rightarrow x$ in a $G$-metric space $(X, G)$, then for any $\varepsilon>0$, there exists a positive integer $N$ such that $G\left(x, x_{n}, x_{m}\right)<\varepsilon$ for all $n, m \geq N$. It was shown in [15] that the G-metric induces a Hausdorff topology and that the convergence, as described in the above definition, is relative to this topology. The topology being Hausdorff, a sequence can converge to at most one point.

Lemma 1 Let $(X, G)$ be a G-metric space, $\left(x_{n}\right)$ be a sequence in $X$ and $x \in X$. Then the following are equivalent:

(1) $\left(x_{n}\right)$ is G-convergent to $x$.

(2) $G\left(x_{n}, x_{n}, x\right) \rightarrow 0$ as $n \rightarrow \infty$.

(3) $G\left(x_{n}, x, x\right) \rightarrow 0$ as $n \rightarrow \infty$.

Lemma 2 If $(X, G)$ is a $G$-metric space, then the following are equivalent:

(1) The sequence $\left(x_{n}\right)$ is G-Cauchy.

(2) For every $\varepsilon>0$, there exists a positive integer $N$ such that $G\left(x_{n}, x_{m}, x_{m}\right)<\varepsilon$ for all $n, m \geq N$.

Lemma 3 Let $(X, G),\left(X^{\prime}, G^{\prime}\right)$ be two G-metric spaces. Then a function $f: X \rightarrow X^{\prime}$ is $G$-continuous at a point $x \in X$ if and only if it is G-sequentially continuous at $x$, that is, if $\left(f x_{n}\right)$ is $G^{\prime}$-convergent to $f x$ whenever $\left(x_{n}\right)$ is G-convergent to $x$.

Definition 6 A $G$-metric space $(X, G)$ is said to be symmetric if

$$
G(x, x, y)=G(x, y, y)
$$

holds for arbitrary $x, y \in X$. If this is not the case, the space is called asymmetric.

To every $G$-metric on the set $X$, a standard metric can be associated by

$$
d_{G}(x, y)=G(x, x, y)+G(x, y, y)
$$

If $G$ is symmetric, then obviously $d_{G}(x, y)=2 G(x, y, y)$, but in the case of an asymmetric G-metric, only

$$
\frac{3}{2} G(x, y, y) \leq d_{G}(x, y) \leq 2 G(x, y, y)
$$

holds for all $x, y \in X$.

The following are some easy examples of $G$-metric spaces.

Example $1(1)$ Let $(X, d)$ be an ordinary metric space. Define $G_{s}$ by

$$
G_{s}(x, y, z)=d(x, y)+d(y, z)+d(x, z)
$$

for all $x, y, z \in X$. Then it is clear that $\left(X, G_{s}\right)$ is a symmetric $G$-metric space. 
(2) Let $X=\{a, b\}$. Define

$$
G(a, a, a)=G(b, b, b)=0, \quad G(a, a, b)=1, \quad G(a, b, b)=2,
$$

and extend $G$ to $X \times X \times X$ by using the symmetry in the variables. Then it is clear that $(X, G)$ is an asymmetric $G$-metric space.

\section{Main results}

First, we define the notion of a generalized weakly cyclic contraction in a $G$-metric space.

Definition 7 Let $(X, G)$ be a $G$-metric space. Let $p$ be a positive integer, $\mathcal{A}_{1}, \mathcal{A}_{2}, \ldots, \mathcal{A}_{p}$ be nonempty subsets of $X$ and $Y=\bigcup_{i=1}^{p} \mathcal{A}_{i}$. An operator $T: Y \rightarrow Y$ is a generalized weakly cyclic contraction if

(I) $Y=\bigcup_{i=1}^{p} \mathcal{A}_{i}$ is a cyclic representation of $Y$ with respect to $T$;

(II) for any $(x, y, z) \in \mathcal{A}_{i} \times \mathcal{A}_{i+1} \times \mathcal{A}_{i+1}, i=1,2, \ldots, p$ (with $\mathcal{A}_{p+1}=\mathcal{A}_{1}$ ),

$$
\psi(G(T x, T y, T z)) \leq \psi(\Theta(x, y, z))-\varphi(\theta(x, y, z))
$$

where

$$
\begin{aligned}
\Theta(x, y, z)= & \max \{G(x, y, z), G(x, T x, T x), G(y, T y, T y), G(z, T z, T z), \\
& \frac{1}{2}[G(x, T y, T y)+G(y, T x, T x)], \frac{1}{2}[G(y, T z, T z)+G(z, T y, T y)], \\
& \frac{1}{2}[G(x, T z, T z)+G(z, T x, T x)], \\
& \left.\frac{1}{3}[G(x, T y, T y)+G(y, T z, T z)+G(z, T x, T x)]\right\}
\end{aligned}
$$

and

$$
\theta(x, y, z)=\max \{G(x, y, z), G(x, T x, T x), G(y, T y, T y), G(z, T z, T z)\}
$$

$\psi:[0,+\infty) \rightarrow[0,+\infty)$ is an altering distance function and $\varphi:[0,+\infty) \rightarrow[0,+\infty)$ is a continuous function with $\varphi(t)=0$ if and only if $t=0$.

Our main result is the following.

Theorem 4 Let $(X, G)$ be a complete $G$-metric space, $p \in \mathbb{N}, \mathcal{A}_{1}, \mathcal{A}_{2}, \ldots, \mathcal{A}_{p}$ be nonempty closed subsets of $X$ and $Y=\bigcup_{i=1}^{p} \mathcal{A}_{i}$. Suppose $T: Y \rightarrow Y$ is a generalized weakly cyclic contraction. Then $T$ has a unique fixed point. Moreover, the fixed point of $T$ belongs to $\bigcap_{i=1}^{p} \mathcal{A}_{i}$.

Proof Let $x_{0} \in \mathcal{A}_{1}$ (such a point exists since $\mathcal{A}_{1} \neq \emptyset$ ). Define the sequence $\left(x_{n}\right)$ in $X$ by

$$
x_{n+1}=T x_{n}, \quad n=0,1,2, \ldots
$$


Without loss of the generality, we can assume that

$$
x_{n} \neq x_{n+1}, \quad \forall n \in \mathbb{N} \cup\{0\} .
$$

We shall prove that

$$
\lim _{n \rightarrow \infty} G\left(x_{n}, x_{n+1}, x_{n+1}\right)=0
$$

By the assumption, $G\left(x_{n}, x_{n+1}, x_{n+1}\right)>0$ for all $n$. From the condition (I), we observe that for all $n$, there exists $i=i(n) \in\{1,2, \ldots, p\}$ such that $\left(x_{n}, x_{n+1}, x_{n+1}\right) \in \mathcal{A}_{i} \times \mathcal{A}_{i+1} \times \mathcal{A}_{i+1}$. Putting $x=x_{n}$ and $y=x_{n+1}=z$ in the condition (II), we have

$$
\begin{aligned}
\Theta\left(x_{n}, x_{n+1}, x_{n+1}\right) & \max \left\{G\left(x_{n}, x_{n+1}, x_{n+1}\right), G\left(x_{n}, T x_{n}, T x_{n}\right), G\left(x_{n+1}, T x_{n+1}, T x_{n+1}\right),\right. \\
& G\left(x_{n+1}, T x_{n+1}, T x_{n+1}\right), \frac{1}{2}\left[G\left(x_{n}, T x_{n+1}, T x_{n+1}\right)+G\left(x_{n+1}, T x_{n}, T x_{n}\right)\right], \\
& \frac{1}{2}\left[G\left(x_{n+1}, T x_{n+1}, T x_{n+1}\right)+G\left(x_{n+1}, T x_{n+1}, T x_{n+1}\right)\right], \\
& \frac{1}{2}\left[G\left(x_{n}, T x_{n+1}, T x_{n+1}\right)+G\left(x_{n+1}, T x_{n}, T x_{n}\right)\right], \\
& \left.\frac{1}{3}\left[G\left(x_{n}, T x_{n+1}, T x_{n+1}\right)+G\left(x_{n+1}, T x_{n+1}, T x_{n+1}\right)+G\left(x_{n+1}, T x_{n}, T x_{n}\right)\right]\right\} \\
= & \max \left\{G\left(x_{n}, x_{n+1}, x_{n+1}\right), G\left(x_{n+1}, x_{n+2}, x_{n+2}\right), \frac{1}{2} G\left(x_{n}, x_{n+2}, x_{n+2}\right),\right. \\
& \left.\frac{1}{3}\left[G\left(x_{n}, x_{n+2}, x_{n+2}\right)+G\left(x_{n+1}, x_{n+2}, x_{n+2}\right)\right]\right\} .
\end{aligned}
$$

By (G5) we have

$$
G\left(x_{n}, x_{n+2}, x_{n+2}\right) \leq G\left(x_{n}, x_{n+1}, x_{n+1}\right)+G\left(x_{n+1}, x_{n+2}, x_{n+2}\right) .
$$

Thus,

$$
\Theta\left(x_{n}, x_{n+1}, x_{n+1}\right)=\theta\left(x_{n}, x_{n+1}, x_{n+1}\right)=\max \left\{G\left(x_{n}, x_{n+1}, x_{n+1}\right), G\left(x_{n+1}, x_{n+2}, x_{n+2}\right)\right\} .
$$

From (3.1) we have

$$
\begin{aligned}
\psi\left(G\left(x_{n+1}, x_{n+2}, x_{n+2}\right)\right)= & \psi\left(G\left(T x_{n}, T x_{n+1}, T x_{n+1}\right)\right) \\
\leq & \psi\left(\Theta\left(x_{n}, x_{n+1}, x_{n+1}\right)\right)-\varphi\left(\theta\left(x_{n}, x_{n+1}, x_{n+1}\right)\right) \\
= & \psi\left(\max \left\{G\left(x_{n}, x_{n+1}, x_{n+1}\right), G\left(x_{n+1}, x_{n+2}, x_{n+2}\right)\right\}\right) \\
& -\varphi\left(\max \left\{G\left(x_{n}, x_{n+1}, x_{n+1}\right), G\left(x_{n+1}, x_{n+2}, x_{n+2}\right)\right\}\right) .
\end{aligned}
$$

We claim that

$$
G\left(x_{n+1}, x_{n+2}, x_{n+2}\right) \leq G\left(x_{n}, x_{n+1}, x_{n+1}\right)
$$


for all $n \geq 0$. Suppose this is not true, that is, there exists an $n_{0} \geq 0$ such that $G\left(x_{n_{0}+1}\right.$, $\left.x_{n_{0}+2}, x_{n_{0}+2}\right)>G\left(x_{n_{0}}, x_{n_{0}+1}, x_{n_{0}+1}\right)$. By the inequality (3.6), for these elements, we have

$$
\begin{aligned}
\psi & \left(G\left(x_{n_{0}+1}, x_{n_{0}+2}, x_{n_{0}+2}\right)\right) \\
\leq & \psi\left(\max \left\{G\left(x_{n_{0}}, x_{n_{0}+1}, x_{n_{0}+1}\right), G\left(x_{n_{0}+1}, x_{n_{0}+2}, x_{n_{0}+2}\right)\right\}\right) \\
& -\varphi\left(\max \left\{G\left(x_{n_{0}}, x_{n_{0}+1}, x_{n_{0}+1}\right), G\left(x_{n_{0}+1}, x_{n_{0}+2}, x_{n_{0}+2}\right)\right\}\right) \\
= & \psi\left(G\left(x_{n_{0}+1}, x_{n_{0}+2}, x_{n_{0}+2}\right)\right)-\varphi\left(G\left(x_{n_{0}+1}, x_{n_{0}+2}, x_{n_{0}+2}\right)\right) .
\end{aligned}
$$

This implies that $\varphi\left(G\left(x_{n_{0}+1}, x_{n_{0}+2}, x_{n_{0}+2}\right)\right)=0$ and by the property of $\varphi$, we have $G\left(x_{n_{0}+1}\right.$, $\left.x_{n_{0}+2}, x_{n_{0}+2}\right)=0$, which contradicts the condition (3.4). Therefore, (3.7) is true and so the sequence $\left(G\left(x_{n}, x_{n+1}, x_{n+1}\right)\right)$ is non-increasing and bounded below. Thus, there exists $\rho \geq 0$ such that

$$
\lim _{n \rightarrow \infty} G\left(x_{n}, x_{n+1}, x_{n+1}\right)=\rho .
$$

Now suppose that $\rho>0$. Taking $n \rightarrow \infty$ in (3.6), then using (3.8) and the continuity of $\psi$ and $\varphi$, we obtain

$$
\psi(\rho) \leq \psi(\rho)-\varphi(\rho)
$$

Therefore, $\varphi(\rho)=0$ and hence $\rho=0$. Thus,

$$
\lim _{n \rightarrow \infty} G\left(x_{n}, x_{n+1}, x_{n+1}\right)=0 .
$$

Next, we show that $\left(x_{n}\right)$ is a G-Cauchy sequence in $X$. Suppose the contrary, that is, $\left(x_{n}\right)$ is not G-Cauchy. Then there exists $\varepsilon>0$ for which we can find two subsequences $\left(x_{m(k)}\right)$ and $\left(x_{n(k)}\right)$ of $\left(x_{n}\right)$ such that $n(k)$ is the smallest index for which

$$
n(k)>m(k)>k, \quad G\left(x_{m(k)}, x_{n(k)}, x_{n(k)}\right) \geq \varepsilon .
$$

This means that

$$
G\left(x_{m(k)}, x_{n(k)-1}, x_{n(k)-1}\right)<\varepsilon .
$$

From (3.9), (3.10) and (G5), we get

$$
\begin{aligned}
\varepsilon & \leq G\left(x_{m(k)}, x_{n(k)}, x_{n(k)}\right) \\
& \leq G\left(x_{m(k)}, x_{n(k)-1}, x_{n(k)-1}\right)+G\left(x_{n(k)-1}, x_{n(k)}, x_{n(k)}\right) \\
& <\varepsilon+G\left(x_{n(k)-1}, x_{n(k)}, x_{n(k)}\right) .
\end{aligned}
$$

Passing to the limit as $k \rightarrow \infty$ and using (3.5), we get

$$
\lim _{k \rightarrow \infty} G\left(x_{m(k)}, x_{n(k)}, x_{n(k)}\right)=\varepsilon
$$


On the other hand, for all $k$, there exists $j(k) \in\{1, \ldots, p\}$ such that $n(k)-m(k)+j(k) \equiv 1[p]$. Then $x_{m(k)-j(k)}$ (for $k$ large enough, $\left.m(k)>j(k)\right)$ and $x_{n(k)}$ lie in different adjacently labeled sets $\mathcal{A}_{i}$ and $\mathcal{A}_{i+1}$ for certain $i \in\{1, \ldots, p\}$. Using (2.1) and (G5), we have

$$
\begin{aligned}
& \left|G\left(x_{m(k)-j(k)}, x_{m(k)-j(k)}, x_{n(k)}\right)-G\left(x_{n(k)}, x_{m(k)}, x_{m(k)}\right)\right| \\
& \quad \leq 2 G\left(x_{m(k)-j(k)}, x_{m(k)-j(k)}, x_{m(k)}\right) \\
& \quad \leq 2 \sum_{l=0}^{j(k)-1} G\left(x_{m(k)-j(k)+l}, x_{m(k)-j(k)+l}, x_{m(k)-j(k)+l+1}\right) \\
& \quad \leq 2 \sum_{l=0}^{p-1} G\left(x_{m(k)-j(k)+l}, x_{m(k)-j(k)+l}, x_{m(k)-j(k)+l+1}\right) \longrightarrow 0
\end{aligned}
$$

as $k \rightarrow \infty$ (from (3.5)), which, by (3.11), implies that

$$
\lim _{k \rightarrow \infty} G\left(x_{m(k)-j(k)}, x_{m(k)-j(k)}, x_{n(k)}\right)=\varepsilon
$$

Using (3.5), we have

$$
\lim _{k \rightarrow \infty} G\left(x_{m(k)-j(k)+1}, x_{m(k)-j(k)+1}, x_{m(k)-j(k)}\right)=0
$$

and

$$
\lim _{k \rightarrow \infty} G\left(x_{n(k)+1}, x_{n(k)+1}, x_{n(k)}\right)=0 .
$$

Again, using (2.1), we get

$$
\begin{aligned}
& \left|G\left(x_{m(k)-j(k)}, x_{m(k)-j(k)}, x_{n(k)+1}\right)-G\left(x_{m(k)-j(k)}, x_{n(k)}, x_{n(k)}\right)\right| \\
& \quad \leq 2 G\left(x_{n(k)}, x_{n(k)+1}, x_{n(k)+1}\right) .
\end{aligned}
$$

Passing to the limit as $k \rightarrow \infty$ in the above inequality, and using (3.13) and (3.12), we get

$$
\lim _{k \rightarrow \infty} G\left(x_{m(k)-j(k)}, x_{m(k)-j(k)}, x_{n(k)+1}\right)=\varepsilon
$$

Similarly, we have

$$
\begin{aligned}
& \left|G\left(x_{n(k)}, x_{m(k)-j(k)+1}, x_{m(k)-j(k)+1}\right)-G\left(x_{m(k)-j(k)}, x_{m(k)-j(k)}, x_{n(k)}\right)\right| \\
& \quad \leq 2 G\left(x_{m(k)-j(k)}, x_{m(k)-j(k)+1}, x_{m(k)-j(k)+1}\right) .
\end{aligned}
$$

Passing to the limit as $k \rightarrow \infty$ and using (3.5) and (3.12), we obtain

$$
\lim _{k \rightarrow \infty} G\left(x_{n(k)}, x_{m(k)-j(k)+1}, x_{m(k)-j(k)+1}\right)=\varepsilon .
$$

Similarly, we have

$$
\lim _{k \rightarrow \infty} G\left(x_{m(k)-j(k)+1}, x_{n(k)+1}, x_{n(k)+1}\right)=\varepsilon
$$


Now, from the definitions of $\Theta(x, y, z)$ and $\theta(x, y, z)$ and from the obtained limits, we have

$$
\begin{aligned}
& \lim _{k \rightarrow \infty} \Theta\left(x_{n(k)+1}, x_{m(k)-j(k)+1}, x_{m(k)-j(k)+1}\right) \\
& \quad=\lim _{k \rightarrow \infty} \theta\left(x_{n(k)+1}, x_{m(k)-j(k)+1}, x_{m(k)-j(k)+1}\right)=\varepsilon .
\end{aligned}
$$

Putting $x=x_{n(k)+1}, y=x_{m(k)-j(k)+1}, z=x_{m(k)-j(k)+1}$ in (II), we obtain

$$
\begin{aligned}
\psi & \left(G\left(x_{n(k)+1}, x_{m(k)-j(k)+1}, x_{m(k)-j(k)+1}\right)\right) \\
= & \psi\left(G\left(T x_{n(k)}, T x_{m(k)-j(k)}, T x_{m(k)-j(k)}\right)\right) \\
\leq & \psi\left(\Theta\left(x_{n(k)+1}, x_{m(k)-j(k)+1}, x_{m(k)-j(k)+1}\right)\right) \\
& -\varphi\left(\theta\left(x_{n(k)+1}, x_{m(k)-j(k)+1}, x_{m(k)-j(k)+1}\right)\right) .
\end{aligned}
$$

Passing to the limit as $k \rightarrow \infty$, utilizing (3.14) and the obtained limits, we get

$$
\psi(\varepsilon) \leq \psi(\varepsilon)-\varphi(\varepsilon)
$$

which is a contradiction if $\varepsilon>0$. We have proved that $\left(x_{n}\right)$ is a $G$-Cauchy sequence in $(X, G)$. Since $(X, G)$ is $G$-complete, there exists $x^{*} \in X$ such that

$$
\lim _{n \rightarrow \infty} x_{n}=x^{*}
$$

We shall prove that

$$
x^{*} \in \bigcap_{i=1}^{p} \mathcal{A}_{i} \text {. }
$$

From condition (I), and since $x_{0} \in \mathcal{A}_{1}$, we have $\left(x_{n p}\right)_{n \geq 0} \subseteq \mathcal{A}_{1}$. Since $\mathcal{A}_{1}$ is closed, from (3.15) we get that $x^{*} \in \mathcal{A}_{1}$. Again, from the condition (I) we have $\left(x_{n p+1}\right)_{n \geq 0} \subseteq \mathcal{A}_{2}$. Since $\mathcal{A}_{2}$ is closed, from (3.15) we get that $x^{*} \in \mathcal{A}_{2}$. Continuing this process, we obtain (3.16).

Now, we shall prove that $x^{*}$ is a fixed point of $T$. Indeed, from (3.16), since for all $n$ there exists $i(n) \in\{1,2, \ldots, p\}$ such that $x_{n} \in \mathcal{A}_{i(n)}$, applying (II) with $x=x_{n}$ and $y=z=x^{*}$, we obtain

$$
\begin{aligned}
\psi\left(G\left(x_{n+1}, T x^{*}, T x^{*}\right)\right) & =\psi\left(G\left(T x_{n}, T x^{*}, T x^{*}\right)\right) \\
& \leq \psi\left(\Theta\left(x_{n}, x^{*}, x^{*}\right)\right)-\varphi\left(\theta\left(x_{n}, x^{*}, x^{*}\right)\right)
\end{aligned}
$$

where

$$
\begin{aligned}
\Theta( & \left.x_{n}, x^{*}, x^{*}\right) \\
= & \max \left\{G\left(x_{n}, x^{*}, x^{*}\right), G\left(x_{n}, T x_{n}, T x_{n}\right), G\left(x^{*}, T x^{*}, T x^{*}\right), G\left(x^{*}, T x^{*}, T x^{*}\right),\right. \\
& \frac{1}{2}\left[G\left(x_{n}, T x^{*}, T x^{*}\right)+G\left(x^{*}, T x_{n}, T x_{n}\right)\right], \frac{1}{2}\left[G\left(x^{*}, T x^{*}, T x^{*}\right)+G\left(x^{*}, T x^{*}, T x^{*}\right)\right], \\
& \frac{1}{2}\left[G\left(x^{*}, T x_{n}, T x_{n}\right)+G\left(x_{n}, T x^{*}, T x^{*}\right)\right],
\end{aligned}
$$




$$
\begin{aligned}
& \left.\frac{1}{3}\left[G\left(x_{n}, T x^{*}, T x^{*}\right)+G\left(x^{*}, T x^{* \prime}, T x^{*}\right)+G\left(x^{*}, T x_{n}, T x_{n}\right)\right]\right\} \\
= & \max \left\{G\left(x_{n}, x^{*}, x^{*}\right), G\left(x_{n}, x_{n+1}, x_{n+1}\right), G\left(x^{*}, T x^{*}, T x^{*}\right),\right. \\
& \frac{1}{2}\left[G\left(x_{n}, T x^{*}, T x^{*}\right)+G\left(x^{*}, x_{n+1}, x_{n+1}\right)\right], \\
& \left.\frac{1}{3}\left[G\left(x_{n}, T x^{*}, T x^{*}\right)+G\left(x^{*}, T x^{*}, T x^{*}\right)+G\left(x^{*}, x_{n+1}, x_{n+1}\right)\right]\right\},
\end{aligned}
$$

and

$$
\theta\left(x_{n}, x^{*}, x^{*}\right)=\max \left\{G\left(x_{n}, x^{*}, x^{*}\right), G\left(x_{n}, x_{n+1}, x_{n+1}\right), G\left(x^{*}, T x^{*}, T x^{*}\right)\right\} .
$$

Passing to the limit as $n \rightarrow \infty$ in the inequality (3.17) and using (3.18), (3.19) and the fact that $G$ is continuous in its variables, we obtain

$$
\psi\left(G\left(x^{\prime \prime}, T x^{\prime \prime}, T x^{*}\right)\right) \leq \psi\left(G\left(x^{*}, T x^{\prime \prime}, T x^{\prime \prime}\right)\right)-\varphi\left(G\left(x^{\prime \prime}, T x^{\prime \prime}, T x^{*}\right)\right) .
$$

This implies that $\varphi\left(G\left(x^{*}, T x^{*}, T x^{*}\right)\right)=0$ and hence $x^{*}=T x^{*}$. Thus, $x^{*}$ is a fixed point of $T$.

Finally, we prove that $x^{\prime \prime}$ is the unique fixed point of $T$. Assume that $y^{\prime \prime}$ is another fixed point of $T$, that is, $T y^{*}=y^{*}$. By the condition (I), this implies that $y^{*} \in \bigcap_{i=1}^{p} \mathcal{A}_{i}$. Then we can apply (II) for $x=x^{*}$ and $y=z=y^{*}$. We obtain

$$
\psi\left(G\left(T x^{*}, T y^{*}, T y^{*}\right)\right) \leq \psi\left(\Theta\left(x^{*}, y^{*}, y^{\prime \prime}\right)\right)-\varphi\left(\theta\left(x^{*}, y^{\prime \prime}, y^{\prime \prime}\right)\right),
$$

where

$$
\begin{aligned}
\Theta\left(x^{*}, y, z\right) & =\max \left\{G\left(x^{*}, y^{*}, y^{*}\right), G\left(x^{*}, T x^{*}, T x^{*}\right), G\left(y^{*}, T y^{*}, T y^{*}\right), G\left(y^{*}, T y^{*}, T y^{*}\right),\right. \\
& \frac{1}{2}\left[G\left(x^{*}, T y^{*}, T y^{*}\right)+G\left(y^{*}, T x^{*}, T x^{*}\right)\right], \frac{1}{2}\left[G\left(y^{*}, T y^{*}, T y^{*}\right)+G\left(y^{*}, T y^{*}, T y^{*}\right)\right], \\
& \frac{1}{2}\left[G\left(x^{*}, T y^{*}, T y^{*}\right)+G\left(y^{*}, T x^{*}, T x^{*}\right)\right], \\
& \left.\frac{1}{3}\left[G\left(x^{*}, T y^{*}, T y^{*}\right)+G\left(y^{*}, T y^{*}, T y^{*}\right)+G\left(y^{*}, T x^{*}, T x^{*}\right)\right]\right\}
\end{aligned}
$$

and

$$
\theta\left(x^{*}, y, z\right)=\max \left\{G\left(x^{*}, y^{*}, y^{*}\right), G\left(x^{*}, T x^{*}, T x^{*}\right), G\left(y^{*}, T y^{*}, T y^{*}\right), G\left(y^{*}, T y^{*}, T y^{*}\right)\right\} .
$$

Since $x^{*}$ and $y^{*}$ are fixed points of $T$, we have from (3.20)-(3.22)

$$
\psi\left(G\left(x^{*}, y^{*}, y^{*}\right)\right) \leq \psi\left(G\left(x^{*}, y^{*}, y^{*}\right)\right)-\varphi\left(G\left(x^{*}, y^{*}, y^{*}\right)\right) .
$$

Hence, $G\left(x^{*}, y^{*}, y^{*}\right)=0$, that is, $x^{*}=y^{*}$. Thus, we have proved the uniqueness of the fixed point. 
Remark 1 Following the proof of Theorem 4, we can derive a similar conclusion if $\Theta(x, y, z)$ and $\theta(x, y, z)$ are replaced, respectively, by

$$
\begin{aligned}
\Theta_{1}(x, y, z)= & \max \{G(x, y, z), G(x, x, T x), G(y, y, T y), G(z, z, T z), \\
& \frac{1}{2}[G(x, y, T y)+G(y, x, T x)], \frac{1}{2}[G(y, z, T z)+G(z, y, T y)], \\
& \frac{1}{2}[G(x, z, T z)+G(z, x, T x)], \\
& \left.\frac{1}{3}[G(x, y, T y)+G(y, z, T z)+G(z, x, T x)]\right\}
\end{aligned}
$$

and

$$
\theta_{1}(x, y, z)=\max \{G(x, y, z), G(x, x, T x), G(y, y, T y), G(z, z, T z)\},
$$

and the condition (3.1) by

$$
\psi(G(T x, T y, T z)) \leq \psi\left(\Theta_{1}(x, y, z)\right)-\varphi\left(\theta_{1}(x, y, z)\right) .
$$

Corollary 1 Let $(X, G)$ be a complete $G$-metric space, $p \in \mathbb{N}, \mathcal{A}_{1}, \mathcal{A}_{2}, \ldots, \mathcal{A}_{p}$ be nonempty closed subsets of $X, Y=\bigcup_{i=1}^{p} \mathcal{A}_{i}$ and $T: Y \rightarrow Y$ such that

(I) $\quad Y=\bigcup_{i=1}^{p} \mathcal{A}_{i}$ is a cyclic representation of $Y$ with respect to $T$;

(II') for any $(x, y, z) \in \mathcal{A}_{i} \times \mathcal{A}_{i+1} \times \mathcal{A}_{i+1}, i=1,2, \ldots, p\left(\right.$ with $\left.\mathcal{A}_{p+1}=\mathcal{A}_{1}\right)$,

$$
\psi(G(T x, T y, T z)) \leq \psi(G(x, y, z))-\varphi(G(x, y, z))
$$

where $\psi:[0,+\infty) \rightarrow[0,+\infty)$ is an altering distance function and $\varphi:[0,+\infty) \rightarrow$ $[0,+\infty)$ is a continuous function with $\varphi(t)=0$ if and only if $t=0$.

Then $T$ has a unique fixed point. Moreover, the fixed point of $T$ belongs to $\bigcap_{i=1}^{p} \mathcal{A}_{i}$.

\section{Examples}

In this section, we furnish some examples to demonstrate the validity of the hypotheses of Theorem 4 .

Example 2 (inspired by [31]) Define a $G$-metric $G$ on the set $X=\{a, b, c\}$ by

$$
\begin{aligned}
& G(x, x, x)=0, \quad x \in X, \\
& G(a, a, b)=2, \\
& G(a, a, c)=G(a, b, b)=G(b, b, c)=3, \\
& G(a, b, c)=G(a, c, c)=G(b, c, c)=4,
\end{aligned}
$$

with symmetry in all variables. Note that $G$ is asymmetric since $G(x, x, y) \neq G(x, y, y)$ whenever $x \neq y$. Let $\mathcal{A}_{1}=\{a, b\}$ and $\mathcal{A}_{2}=\{a, c\}$ and consider the mapping $T: X \rightarrow X$ given by 
$T a=T b=a$ and $T c=b$. Obviously, $\mathcal{A}_{1} \cup \mathcal{A}_{2}=X$ is a cyclic representation of $X$ with respect to $T$.

Take $\psi(t)=t^{2}$ and $\varphi(t)=t$ and denote $L=\psi(G(T x, T y, T z)), R=\psi(\Theta(x, y, z))-\varphi(\theta(x$, $y, z)$ ). The following table shows that the contractive condition (3.1) is fulfilled whenever $(x, y, z) \in \mathcal{A}_{1} \times \mathcal{A}_{2} \times \mathcal{A}_{2}$ or $(x, y, z) \in \mathcal{A}_{2} \times \mathcal{A}_{1} \times \mathcal{A}_{1}$.

$\begin{array}{cccccc}(x, y, z) & G(T x, T y, T z) & \Theta(x, y, z) & \theta(x, y, z) & L & R \\ (a, a, a) & 0 & 0 & 0 & 0 & 0 \\ (a, a, b) & 0 & 2 & 2 & 0 & 2 \\ (a, a, c) & 2 & 3 & 3 & 4 & 6 \\ (a, b, b) & 0 & 3 & 3 & 0 & 6 \\ (a, b, c) & 2 & 4 & 4 & 4 & 12 \\ (a, c, c) & 3 & 4 & 4 & 9 & 12 \\ (b, b, c) & 2 & 3 & 3 & 4 & 6 \\ (b, c, c) & 3 & 4 & 4 & 9 & 12\end{array}$

Hence, all the conditions of Theorem 4 are fulfilled and it follows that $T$ has a unique fixed point $a \in \mathcal{A}_{1} \cap \mathcal{A}_{2}$.

Example 3 Let $X=\mathbb{R}^{3}$ and let $G: X^{3} \rightarrow \mathbb{R}^{+}$be given as

$$
G\left(\left(x_{1}, y_{1}, z_{1}\right),\left(x_{2}, y_{2}, z_{2}\right),\left(x_{3}, y_{3}, z_{3}\right)\right)=\sum_{i=1}^{3}\left[\left|x_{i}-x_{i+1}\right|+\left|y_{i}-y_{i+1}\right|+\left|z_{i}-z_{i+1}\right|\right],
$$

where $\left(x_{4}, y_{4}, z_{4}\right)=\left(x_{1}, y_{1}, z_{1}\right)$. It is easy to see that $(X, G)$ is a complete $G$-metric space. Consider the following closed subsets of $X$ :

$$
\begin{array}{ll}
\mathcal{A}_{1}=\{(a, 0,0): 0 \leq a \leq 1\}, & \mathcal{A}_{2}=\{(0, b, 0): 0 \leq b \leq 1\}, \\
\mathcal{A}_{3}=\{(0,0, c): 0 \leq c \leq 1\}, & Y=\mathcal{A}_{1} \cup \mathcal{A}_{2} \cup \mathcal{A}_{3}
\end{array}
$$

and the mapping $T: Y \rightarrow Y$ given by

$$
T(a, 0,0)=\left(0, \frac{a}{2}, 0\right), \quad T(0, b, 0)=\left(0,0, \frac{b}{4}\right), \quad T(0,0, c)=\left(\frac{c}{8}, 0,0\right) .
$$

It is clear that $Y=\mathcal{A}_{1} \cup \mathcal{A}_{2} \cup \mathcal{A}_{3}$ is a cyclic representation of $Y$ with respect to $T$. We will check that $T$ satisfies the contraction condition (II).

Take $\psi(t)=t^{2}$ (which is an altering distance function) and a continuous function $\varphi(t)=$ $\frac{3 t^{2}}{4}$ such that $\varphi(0)=0$. Let, e.g., $(x, y, z) \in \mathcal{A}_{1} \times \mathcal{A}_{2} \times \mathcal{A}_{2}$ (the other two cases are treated analogously), and let $x=(a, 0,0), y=(0, b, 0), z=(0, c, 0)$. Without loss of generality, we can assume that $b \geq c$. Then $T x=\left(0, \frac{a}{2}, 0\right), T y=\left(0,0, \frac{b}{4}\right), T z=\left(0,0, \frac{c}{4}\right)$ and

$$
\begin{aligned}
& G(T x, T y, T z)=a+\frac{b}{2}, \quad G(x, y, z)=2(a+b), \\
& G(x, T x, T x)=3 a, \quad G(y, T y, T y)=\frac{5}{2} b, \quad G(z, T z, T z)=\frac{5}{2} c,
\end{aligned}
$$




$$
\begin{aligned}
& \frac{1}{2}[G(x, T y, T y)+G(y, T x, T x)]=\frac{1}{2}\left(2 a+\frac{b}{2}+2\left|b-\frac{a}{2}\right|\right), \\
& \frac{1}{2}[G(y, T z, T z)+G(z, T y, T y)]=\frac{5}{4}(b+c), \\
& \frac{1}{2}[G(x, T z, T z)+G(z, T x, T x)]=\frac{1}{2}\left(2 a+\frac{c}{2}+2\left|c-\frac{a}{2}\right|\right), \\
& \frac{1}{3}(G(x, T y, T y)+G(y, T z, T z)+G(z, T x, T x))=\frac{1}{3}\left(2 a+\frac{5}{2} b+\frac{c}{2}+2\left|c-\frac{a}{2}\right|\right) .
\end{aligned}
$$

Hence,

$$
\begin{aligned}
\psi(G(T x, T y, T z)) & =\left(a+\frac{b}{2}\right)^{2} \leq \frac{1}{4}(2(a+b))^{2} \\
& =\frac{1}{4} \psi(G(x, y, z)) \\
& \leq \psi(\Theta(x, y, z))-\varphi(\theta(x, y, z)) .
\end{aligned}
$$

Thus, the conditions of Theorem 4 are fulfilled and $T$ has a unique fixed point $(0,0,0) \in$ $\bigcap_{i=1}^{3} \mathcal{A}_{i}$.

\section{An application to boundary value problems}

In this section, we present another example where Theorem 4 and its corollaries can be applied. The example is inspired by [36].

We study the existence of a solution for the following two-point boundary value problem for a second-order differential equation:

$$
\left\{\begin{array}{l}
\frac{d^{2} u}{d t^{2}}=K(t, u(t)), \quad t \in[0,1], u \in[0, \infty), \\
u(0)=u(1)=0
\end{array}\right.
$$

where $K:[0,1] \times \mathbb{R}^{+} \rightarrow \mathbb{R}^{+}$is a continuous function. This problem is equivalent to the integral equation

$$
u(t)=\int_{0}^{1} \mathcal{G}(t, s) K(s, u(s)) d s, \quad \text { for } t \in[0,1]
$$

where $\mathcal{G}(t, s)$ is the Green function

$$
\mathcal{G}(t, s)= \begin{cases}t(1-s), & 0 \leq s<t \leq 1 \\ s(1-t), & 0 \leq t<s \leq 1\end{cases}
$$

Denote by $X=C\left([0,1], \mathbb{R}^{+}\right)$the set of non-negative continuous real functions on $[0,1]$. We endow $X$ with the $G$-metric

$$
G(u, v, w)=\max _{t \in[0,1]}|u(t)-v(t)|+\max _{t \in[0,1]}|u(t)-w(t)|+\max _{t \in[0,1]}|v(t)-w(t)|
$$

for $u, v, w \in X$. Then $(X, G)$ is a complete $G$-metric space. 
Consider the self-map $T: X \rightarrow X$ defined by

$$
\operatorname{Tu}(t)=\int_{0}^{1} \mathcal{G}(t, s) K(s, u(s)) d s, \quad \text { for } t \in[0,1] .
$$

Clearly, $u^{\prime \prime}$ is a solution of (5.2) if and only if $u^{\prime \prime}$ is a fixed point of $T$.

We will prove the existence and uniqueness of the fixed point of $T$ under the following conditions.

(A) $K(s, \cdot)$ is a non-increasing function for any fixed $s \in[0,1]$, that is,

$$
x, y \in \mathbb{R}^{+}, x \geq y \quad \Longrightarrow \quad K(s, x) \leq K(s, y) .
$$

(B) $|K(s, x)-K(s, y)| \leq|x-y|$ for all $s \in[0,1]$ and $x, y \in \mathbb{R}^{+}$.

(C) There exist $\alpha, \beta \in X$ such that $\alpha(t) \leq \beta(t)$ for $t \in[0,1]$ and that

$$
T \alpha(t) \leq \beta(t) \quad \text { and } \quad T \beta(t) \geq \alpha(t) \quad \text { for } t \in[0,1] .
$$

Theorem 5 Under the conditions (A)-(C), equation (5.2) has a unique solution $u^{*} \in X$ and it belongs to $\mathcal{C}=\{u \in X: \alpha(t) \leq u(t) \leq \beta(t), t \in[0,1]\}$.

Proof In order to prove the existence of a (unique) fixed point of $T$, we construct closed subsets $\mathcal{A}_{1}$ and $\mathcal{A}_{2}$ of $X$ as follows:

$$
\mathcal{A}_{1}=\{u \in X: u(t) \leq \beta(t), t \in[0,1]\}
$$

and

$$
\mathcal{A}_{2}=\{u \in X: u(t) \geq \alpha(t), t \in[0,1]\} .
$$

We shall prove that

$$
T\left(\mathcal{A}_{1}\right) \subseteq \mathcal{A}_{2} \quad \text { and } \quad T\left(\mathcal{A}_{2}\right) \subseteq \mathcal{A}_{1} .
$$

Let $u \in \mathcal{A}_{1}$, that is,

$$
u(s) \leq \beta(s), \quad \text { for all } s \in[0,1] .
$$

Since $\mathcal{G}(t, s) \geq 0$ for all $t, s \in[0,1]$, we deduce from (A) and (C) that

$$
\int_{0}^{1} \mathcal{G}(t, s) K(s, u(s)) d s \geq \int_{0}^{1} \mathcal{G}(t, s) K(s, \beta(s)) d s \geq \alpha(t)
$$

for all $t \in[0,1]$. Then we have $T u \in \mathcal{A}_{2}$. Similarly, the other inclusion is proved. Hence, $Y=\mathcal{A}_{1} \cup \mathcal{A}_{2}$ is a cyclic representation of $Y$ with respect to $T$.

Finally, we will show that, for each $u \in \mathcal{A}_{1}$ and $v, w \in \mathcal{A}_{2}$, one has

$$
\psi(G(T u, T v, T w)) \leq \psi(G(u, v, w))-\varphi(G(u, v, w))
$$

for $\psi(t)=t$ and $\varphi(t)=\frac{7}{8} t$. 
To this end, let $u \in \mathcal{A}_{1}$ and $(v, w) \in \mathcal{A}_{2} \times \mathcal{A}_{2}$. Therefore, by (B) we deduce that for each $t \in[0,1]$,

$$
\begin{aligned}
& G(T u, T v, T w) \\
&=\max _{t \in[0,1]}|T u(t)-T v(t)|+\max _{t \in[0,1]}|T u(t)-T w(t)|+\max _{t \in[0,1]}|T v(t)-T w(t)| \\
&=\max _{t \in[0,1]} \int_{0}^{1} \mathcal{G}(t, s)|K(s, u(s))-K(s, v(s))| d s \\
& \quad+\max _{t \in[0,1]} \int_{0}^{1} \mathcal{G}(t, s)|K(s, u(s))-K(s, w(s))| d s \\
& \quad+\max _{t \in[0,1]} \int_{0}^{1} \mathcal{G}(t, s)|K(s, v(s))-K(s, w(s))| d s \\
& \leq \max _{t \in[0,1]} \int_{0}^{1} \mathcal{G}(t, s)(|u(s)-v(s)|+|u(s)-w(s)|+|v(s)-w(s)|) d s \\
& \leq G(u, v, w) \max _{t \in[0,1]} \int_{0}^{1} \mathcal{G}(t, s) d s .
\end{aligned}
$$

It is easy to verify that

$$
\int_{0}^{1} \mathcal{G}(t, s) d s=\frac{-t^{2}}{2}+\frac{t}{2} \leq \frac{1}{8}, \quad \text { for } 0 \leq t \leq 1
$$

With these facts, the inequality (5.3) gives us

$$
G(T u, T v, T w) \leq \frac{1}{8} G(u, v, w)
$$

and we have

$$
\psi(G(T u, T v, T w)) \leq \psi(G(u, v, w))-\varphi(G(u, v, w)) .
$$

Using the same technique, we can show that the above inequality also holds if we take $(u, v, w) \in \mathcal{A}_{2} \times \mathcal{A}_{1} \times \mathcal{A}_{1}$. Thus, $T$ satisfies the contractive condition of Corollary 1 .

Consequently, by Corollary $1, T$ has a unique fixed point $u^{\prime \prime} \in \mathcal{A}_{1} \cap \mathcal{A}_{2}$, that is, $u^{\prime \prime} \in \mathcal{C}$ is the unique solution to (5.2).

\section{Competing interests}

The authors declare that they have no competing interests.

\section{Authors' contributions}

All authors contributed equally and significantly in writing this paper. All authors read and approved the final manuscript.

\section{Author details}

'Department of Mathematics, Disha Institute of Management and Technology, Satya Vihar, Vidhansabha-Chandrakhuri Marg, Mandir Hasaud, Raipur, Chhattisgarh 492101, India. ²Faculty of Mechanical Engineering, University of Belgrade, Kraljice Marije 16, Beograd, 11120, Serbia. ${ }^{3}$ Faculty of Mathematics, University of Belgrade, Studentski trg 16, Beograd, 11000 , Serbia. 


\section{Acknowledgements}

The authors thank the referees for their valuable comments that helped them to correct the first version of the manuscript. The second and third authors are thankful to the Ministry of Science and Technological Development of Serbia.

Received: 20 August 2012 Accepted: 25 November 2012 Published: 17 December 2012

\section{References}

1. Banach, S: Sur les opérations dans les ensembles abstraits et leur application aux equations integrales. Fundam. Math. 3, 133-181 (1922)

2. Nashine, HK: New fixed point theorems for mappings satisfying generalized weakly contractive condition with weaker control functions. Ann. Pol. Math. 104, 109-119 (2012)

3. Kirk, WA, Srinavasan, PS, Veeramani, P: Fixed points for mapping satisfying cyclical contractive conditions. Fixed Point Theory 4, 79-89 (2003)

4. Boyd, DW, Wong, SW: On nonlinear contractions. Proc. Am. Math. Soc. 20, 458-464 (1969)

5. Caristi, J: Fixed point theorems for mappings satisfying inwardness conditions. Trans. Am. Math. Soc. 215, 241-251 (1976)

6. Rus, IA: Cyclic representations and fixed points. Ann. "Tiberiu Popoviciu" Sem. Funct. Equ. Approx. Convexity 3, $171-178(2005)$

7. Di Bari, C, Suzuki, T, Vetro, C: Best proximity points for cyclic Meir-Keeler contractions. Nonlinear Anal. 69, 3790-3794 (2008)

8. Păcurar, M, Rus, IA: Fixed point theory for cyclic $\varphi$-contractions. Nonlinear Anal. 72, 2683-2693 (2010)

9. Piatek, B: On cyclic Meir-Keeler contractions in metric spaces. Nonlinear Anal. 74, 35-40 (2011)

10. Karapinar, E: Fixed point theory for cyclic weaker $\phi$-contraction. Appl. Math. Lett. 24, 822-825 (2011)

11. Karapinar, E, Sadarangani, K: Fixed point theory for cyclic $(\phi-\varphi)$-contractions. Fixed Point Theory Appl. 2011, Art. ID 69 (2011)

12. Nashine, HK: Cyclic generalized $\psi$-weakly contractive mappings and fixed point results with applications to integral equations. Nonlinear Anal. 75, 6160-6169 (2012)

13. Chen, CM: Fixed point theorems for cyclic Meir-Keeler type mappings in complete metric spaces. Fixed Point Theory Appl. 2012, Art. ID 41 (2012)

14. Mustafa, Z, Sims, B: Some remarks concerning D-metric spaces. In: Proc. Int. Conf. on Fixed Point Theory and Applications, Valencia, Spain, July 2003, pp. 189-198 (2003)

15. Mustafa, Z, Sims, B: A new approach to generalized metric spaces. J. Nonlinear Convex Anal. 7(2), $289-297$ (2006)

16. Mustafa, Z, Obiedat, $\mathrm{H}$, Awawdeh, F: Some fixed point theorem for mapping on complete G-metric spaces. Fixed Point Theory Appl. 2008, Art. ID 189870 (2008)

17. Mustafa, Z, Sims, B: Fixed point theorems for contractive mapping in complete G-metric spaces. Fixed Point Theory Appl. 2009, Art. ID 917175 (2009)

18. Mustafa, Z, Shatanawi, W, Bataineh, M: Existence of fixed point results in G-metric spaces. Int. J. Math. Anal. 2009, Art. ID 283028 (2009)

19. Chugh, R, Kadian, T, Rani, A, Rhoades, BE: Property P in G-metric spaces. Fixed Point Theory Appl. 2010, Art. ID 401684 (2010)

20. Shatanawi, W: Fixed point theory for contractive mappings satisfying $\Phi$-maps in G-metric spaces. Fixed Point Theory Appl. 2010, Art. ID 181650 (2010)

21. Mustafa, Z, Khandagjy, M, Shatanawi, W: Fixed point results on complete G-metric spaces. Studia Sci. Math. Hung. 48 304-319 (2011)

22. Mustafa, Z, Aydi, H, Karapinar, E: On common fixed points in G-metric spaces using (E.A) property. Comput. Math Appl. 64, 1944-1956 (2012)

23. Mustafa, Z: Some new common fixed point theorems under strict contractive conditions in G-metric spaces. J. Appl. Math. 2012, Art. ID 248937 (2012)

24. Kaewcharoen, $A$ : Common fixed point theorems for contractive mappings satisfying $\Phi$-maps in $G$-metric spaces Banach J. Math. Anal. 6, 101-111 (2012)

25. Kadelburg, Z, Nashine, HK, Radenović, S: Common coupled fixed point results in partially ordered G-metric spaces. Bull. Math. Anal. Appl. 4, 51-63 (2012)

26. Nashine, HK, Kadelburg, Z, Pathak, RP, Radenović, S: Coincidence and fixed point results in ordered G-cone metric spaces. Math. Comput. Model. 57, 701-709 (2013)

27. Khan, MS, Swaleh, M, Sessa, S: Fixed point theorems by altering distances between the points. Bull. Aust. Math. Soc 30, 1-9 (1984)

28. Rhoades, BE: Some theorems on weakly contractive maps. Nonlinear Anal. 47, 2683-2693 (2001)

29. Dutta, PN, Choudhury, BS: A generalisation of contraction principle in metric spaces. Fixed Point Theory Appl. 2008, Art. ID 406368 (2008)

30. Radenović, S, Kadelburg, Z, Jandrlić, D, Jandrlić, A: Some results on weak contraction maps. Bull. Iran. Math. Soc. (to appear)

31. Choudhury, BS, Maity, P: Coupled fixed point results in generalized metric spaces. Math. Comput. Model. 54, 73-79 (2011)

32. Shatanawi, W, Mustafa, Z: On coupled random fixed point results in partially ordered metric spaces. Mat. Vesn. 64, 139-146 (2012)

33. Mustafa, Z, Aydi, H, Karapinar, E: Mixed g-monotone property and quadruple fixed point theorems in partially ordered metric spaces. Fixed Point Theory Appl. 2012, Art. ID 71 (2012)

34. Shatanawi, WA, Postolache, M, Mustafa, Z, Tahat, N: Theorems of Boyd-Wong type contractions in ordered metric spaces. Abstr. Appl. Anal. 2012, Art. ID 359054 (2012)

35. Karapinar, E, Shatanawi, W, Mustafa, Z: Quadruple point theorems under nonlinear contractive conditions in partially ordered metric spaces. J. Appl. Math. 2012, Art. ID 951912 (2012)

36. Harjani, J, Sadarangani, K: Generalized contractions in partially ordered metric spaces and applications to ordinary differential equations. Nonlinear Anal. 72, 1188-1197 (2010) 
doi:10.1186/1687-1812-2012-227

Cite this article as: Nashine et al.: Nonlinear cyclic weak contractions in G-metric spaces and applications to boundary value problems. Fixed Point Theory and Applications 2012 2012:227.

Submit your manuscript to a SpringerOpen ${ }^{\circ}$ journal and benefit from:

- Convenient online submission

- Rigorous peer review

- Immediate publication on acceptance

- Open access: articles freely available online

- High visibility within the field

- Retaining the copyright to your article

Submit your next manuscript at $\gg$ springeropen.com 